\title{
Minute Times gram Per Milliliter Per Milligram Per Gram
}

National Cancer Institute

\section{Source}

National Cancer Institute. Minute Times gram Per Milliliter Per Milligram Per Gram. NCI

Thesaurus. Code C117940.

Minute times gram per milliliter, divided by milligram per gram. 